\title{
EMPODERAMIENTO DOCENTE EN EL ÁMBITO DE LAS ALTAS CAPACIDADES INTELECTUALES. MITOS Y CREENCIAS EN LOS DOCENTES DE EDUCACIÓN PRIMARIA
}

\author{
Emilio López Parra \\ María Inés Martín García \\ Ascensión Palomares Ruiz \\ Universidad de Castilla La Mancha
}

\begin{abstract}
RESUMEN: El alumnado con altas capacidades intelectuales tiene necesidades educativas que por diversas causas no siempre son atendidas adecuadamente. En esta compleja situación se enmarca esta investigación cuya finalidad es la de conocer cuáles son las percepciones de los docentes de Educación Primaria sobre aspectos tan relevantes como el rendimiento, conductas y habilidades sociales, género y respuesta educativa que satisfaga sus necesidades educativas. Se indaga sobre la formación de estos docentes, y la influencia de estereotipos y mitos sobre las altas capacidades. Se utiliza una metodología mixta, que en su enfoque cuantitativo analiza las percepciones de 343 docentes mediante un cuestionario, y desde la perspectiva cualitativa, recoge las preferencias sobre la respuesta educativa más eficaz para este alumnado, mediante una entrevista estructurada a 6 docentes. Los resultados muestran que todavía continúan arraigados algunos de los mitos y estereotipos más comunes sobre este alumnado, existiendo deficiencias importantes en el plano formativo y que la respuesta educativa debería de ser más individualizada según las necesidades de cada alumno. Esto nos lleva a concluir que es necesario establecer programas formativos en altas capacidades para los docentes, como medida fundamental para mejorar la vida académica y personal del alumnado con altas capacidades intelectuales.
\end{abstract}

PALABRAS CLAVE: Altas capacidades intelectuales, Educación Primaria, formación docente, educación inclusiva, atención educativa. 


\title{
EDUCATIONAL EMPOWERMENT IN THE FIELD OF HIGH INTELLECTUAL CAPACITIES. MYTHS AND BELIEFS IN THE PRIMARY EDUCATION TEACHERS
}

\begin{abstract}
Students with high intellectual abilities have educational needs that for various reasons are not always adequately addressed. This research is defined in this complex situation and its purpose is to know what the perceptions of Primary Education teachers in relation to such important aspects as performance, social behaviours and skills, gender and the most appropriate educational response to meet their educational needs. It looks into these teachers' training, and the influence of this training in the presence of stereotypes and myths about high abilities. A mixed methodology is used, whose quantitative approach analyze the perceptions and beliefs of 343 teachers through a questionnaire and, from a qualitative perspective, it picks the preferences on the most effective educational response for these students through a structured interview on 6 primary education teachers. The results show that some of the most common myths and stereotypes about these students are still rooted, that there are still important deficiencies in the training level and that the educational response should be more individualized according to the needs of each student. This leads us to conclude that it is necessary to establish training programs for teachers, as a fundamental element to improve the academic and personal life of students with high intellectual abilities.
\end{abstract}

KEYWORDS: High intellectual capacities, Primary Education, teacher training, inclusive education, educational attention.

Recibido: 10/01/2019

Aceptado: 11/06/2019

Correspondencia: María Inés Martín García, Universidad de Castilla La Mancha, Plaza de la Universidad 3, 02071 Albacete. Email: Marialnes.Martin@uclm.es.

\section{INTRODUCCIÓN}

La comunidad educativa debe velar para que la atención a la diversidad sea además de un derecho, un hecho constatable en todas las aulas de nuestro país. Que cada alumno reciba la atención educativa que necesita ha de ser una prioridad que ha de trasladarse del plano teórico a la siempre compleja realidad del aula y, en este sentido, adquiere especial relevancia el alumnado con altas capacidades intelectuales que por su complejidad necesita una atención educativa específica para que pueda desarrollar al máximo sus potencialidades (Nolla, 2012).

Los datos estadísticos del curso 2015/2016 según el Ministerio de Educación y Formación Profesional, nos indican que, el porcentaje de alumnos de Educación Primaria diagnosticados en España, como alumnado con altas capacidades intelectuales se sitúa en el 0,42\%, quedando muy por debajo, de la estimación mínima que se establece en 
el informe Marland (1972) de entre un $3 \%$ y un $5 \%$, y mucho más lejos del $10 \%$ que establece Gagné, o del 20\% que indica Renzulli, según señala Tourón (2015)

Estos datos nos llevan a entender la complicada situación de la inmensa mayoría de estos alumnos, que no están recibiendo la atención educativa que necesitan (Hernández y Gutiérrez, 2014), lo que significa que un gran porcentaje de ellos se verán abocados al fracaso escolar, y les hace ser, en muchas ocasiones, los grandes olvidados dentro de los alumnos con necesidades específicas de apoyo educativo (González y Palomares, 2016). Para reconducir esta situación es fundamental que emerja con fuerza la figura del docente. Pero un docente capaz de intervenir con eficacia en el momento crucial, es decir, de guiar y ayudar al alumnado con altas capacidades para una integración social exitosa (Branda y Porta, 2012). Un docente debidamente formado para poder intervenir en el proceso de identificación de estos alumnos con altas capacidades y también para poder proporcionar una respuesta educativa adecuada, eficaz y que permita a estos alumnos desarrollar al máximo sus potencialidades.

El hecho de establecer una definición concreta sobre las altas capacidades es una misión compleja, debido a las distintas perspectivas que de estas se han hecho (Agudo, 2017), por lo que la ausencia de una definición de consenso del concepto de superdotación o del de altas capacidades intelectuales (Sánchez Manzano, 2010) genera cierta confusión, y provoca que, como señala Carman (2013), se utilicen múltiples modelos, cada uno con unos determinados criterios, a la hora de identificar a este tipo de alumnado.

Esta cuestión incide en que la detección y la identificación del alumnado con altas capacidades intelectuales sea el paso más complejo y sobre el que más trabajo queda por hacer, ya que es el que inicia todo el procedimiento para proporcionar una adecuada respuesta educativa y alcanzar un eficaz desarrollo de las potencialidades del alumnado. Como indica Sánchez Manzano (2009), el fin de la identificación es proporcionar al alumnado con altas capacidades la respuesta educativa que mejor se adapte a sus necesidades.

Todavía a día de hoy, los procesos para la detección y diagnóstico del alumnado con altas capacidades no están instaurados de forma habitual o protocolaria en los centros educativos (Wellisch y Brown, 2012), o si lo están, prevalece en ellos la medida de la capacidad intelectual como condición previa para la identificación de estos estudiantes (Carma, 2013; Hernández y Gutiérrez, 2014). En esta línea, Calero y García (2011) señalan que en España no hay establecido ningún tipo de programa específico para el alumnado con altas capacidades, no habiendo ni siquiera consenso sobre la respuesta educativa más adecuada que se le debe proporcionar.

En relación a los maestros y maestras, hay que considerarlos como elementos claves para favorecer la situación educativa del este tipo de alumnado, ya sea por su actuación dentro del aula o bien por la adopción de medidas como la aceleración de algunas materias o cursos completos (Wellisch y Brown, 2012), de tal forma que como apunta Reis y Renzulli (2010), una deficiente formación docente en el ámbito de las altas capacidades origina en este alumnado bajo rendimiento e incluso abandono escolar. 
Es fundamental que el profesorado esté formado en altas capacidades para asegurar el éxito de cualquier medida educativa adoptada, ya que aquellos docentes sin formación suelen mostrarse desinteresados y con rechazo hacia este tipo de alumnado (Alonso, 2008). Además, es preciso que esta formación sea de calidad, ya que suelen percibir que no han adquirido la suficiente formación para atender educativamente a este alumnado (Castro, 2005; Peña del Agua, Martínez, Velázquez, Barriales y López, 2003; Muglia y Tonete, 2016).

Numerosas investigaciones han hallado que entre los distintos motivos por los que los docentes no implementan estrategias adecuadas a las necesidades del alumnado con altas capacidades, destaca el de respaldar sus acciones educativas basándose en mitos y estereotipos, obviando los estudios científicos en este ámbito (Rodrigues y De Souza, 2012).

Los numerosos los mitos y creencias vinculados a las altas capacidades suelen influenciar a los docentes y familias moldeando una determinada actitud en este ámbito, con el consiguiente obstáculo en la identificación, evaluación, atención educativa y el propio desarrollo de este alumnado (Martínez y Guirado, 2010).

Como señalan Baudson y Preckel (2016), los estereotipos que tienen los docentes no solo impiden que el alumnado con altas capacidades sea identificado y por tanto que no reciba la respuesta educativa que necesita, sino que pueden generar trastornos en el desarrollo de su personalidad. Además, incide en resaltar que no solamente los estereotipos que tienen connotación negativa son perjudiciales, sino también aquellos estereotipos y mitos que tienen un carácter positivo, ya que sitúan a estos alumnos bajo presión.

Sobre estas cuestiones se plantea el objetivo general de conocer la percepción y competencias que tienen los docentes de Educación Primaria sobre las altas capacidades intelectuales, así como la respuesta educativa que se le proporciona a este alumnado.

\section{Método}

La metodología utilizada en esta investigación es de tipo mixto, con un diseño incrustado concurrente de modelo dominante (DIAC) (Hernández, Fernández y Baptista, 2014).

Razonadamente, para el cumplimiento de los objetivos planteados, se ha considerado realizar una investigación siguiendo una metodología cuantitativa de carácter descriptivo (principal), con un componente de carácter cualitativo (secundario).

\section{Población y muestra}

La población en esta investigación ha estado constituida por maestros y maestras de Educación Primaria en activo durante el curso 2017-2018 en los centros escolares de Albacete. 
La selección muestral en el enfoque cuantitativo ha seguido un método no probabilístico, de tipo por conveniencia. La elección de los centros escolares cuyos docentes integrarían la muestra del estudio se ha realizado atendiendo a los criterios de cercanía y accesibilidad, por lo que los resultados no pretenden ser representativos de la población y no podrán generalizarse.

De esta forma, la muestra está constituida por 343 maestros y maestras de Educación Primaria, pertenecientes a 15 colegios de la ciudad de Albacete. De los 343 docentes, el $74,3 \%$ eran mujeres $(n=255)$ y el $25,6 \%$ hombres $(n=88)$. La experiencia docente que tienen los componentes muestrales es amplia, ya que el 95,2\% manifiesta tener una experiencia de más de 11 años, teniendo un 67,6\% más de 20 años de experiencia docente. Del total de la muestra, el $66,7 \%$ no ha tenido nunca alumnado diagnosticado con altas capacidades en sus aulas. Respecto a la formación, el 75,2\% de los docentes no ha adquirido ningún tipo de formación específica vinculada con las altas capacidades, el 24,8\% restante, afirma haberla adquirido fundamentalmente durante sus estudios en la universidad $(46,7 \%)$, a través de cursos de formación $(36,7 \%)$.

La selección del grupo muestral en el enfoque cualitativo se realizó mediante un método no probabilístico, de tipo por conveniencia, constituida por seis docentes, tres mujeres y tres hombres, con distintos grados de experiencia docente.

\section{Instrumento}

En el método cuantitativo se ha utilizado la técnica de la encuesta, siendo el instrumento elegido para la recogida de datos el cuestionario, el cual consta de 25 ítems de respuesta cerrada con escala tipo Likert de cinco grados, aplicándose de manera autoadministrada, en contexto individual y por envío a través de la plataforma. El cuestionario utilizado es el de González y Palomares (2016), por lo que está debidamente validado, mostrando una fiabilidad de 0,737 (Alfa de Cronbach) en la escala de percepciones y estereotipos, y de 0,875 (Alfa de Cronbach), la escala sobre la respuesta educativa, consideradas alta muy alta respectivamente, según Palella y Martins (2012).

En el método cualitativo se ha utilizado la técnica de la entrevista, utilizando como instrumento un guion de entrevista formalizada, de tipo entrevista monotemática (Palella y Martins, 2012). Consta de 5 preguntas vinculadas con la respuesta educativa al alumnado con altas capacidades intelectuales

\section{Resultados}

Se obtuvieron resultados relativos a las percepciones y estereotipos vinculados con diferentes aspectos de las altas capacidades intelectuales, concretamente sobre cuestiones de género, rendimiento y éxito escolar, sobre las conductas de este alumnado y sobre la atención educativa.

En aspectos de género, el 10,5\% de los docentes encuestados considera que es más fácil identificar como alumnado con altas capacidades a los niños que a las niñas, mientras que un 61,9\% muestra su desacuerdo con esta afirmación (Figura 1). 


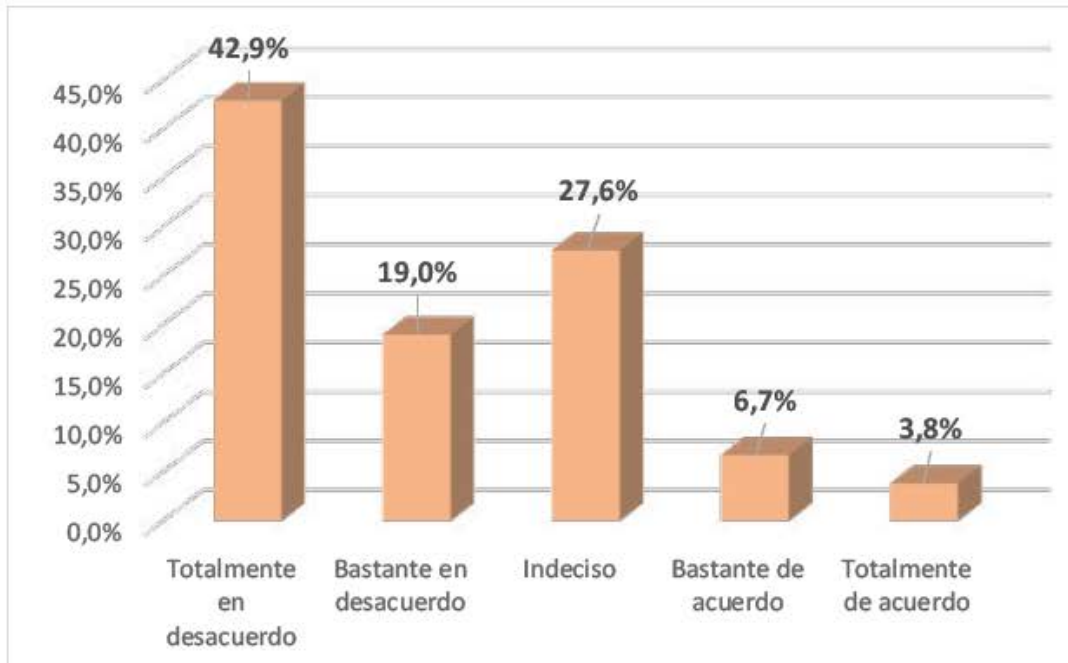

Figura 1. Grado de acuerdo sobre la mayor facilidad de identificar a los niños con altas capacidades que a las niñas con altas capacidades. Fuente: Elaboración propia

Sobre si este tipo de alumnado tiene buen rendimiento escolar generalmente, el $22,8 \%$ de los docentes encuestados rechaza esta idea, mostrándose indecisos el $46,7 \%$, y estando de acuerdo el $30,5 \%$ (Figura 2 ).

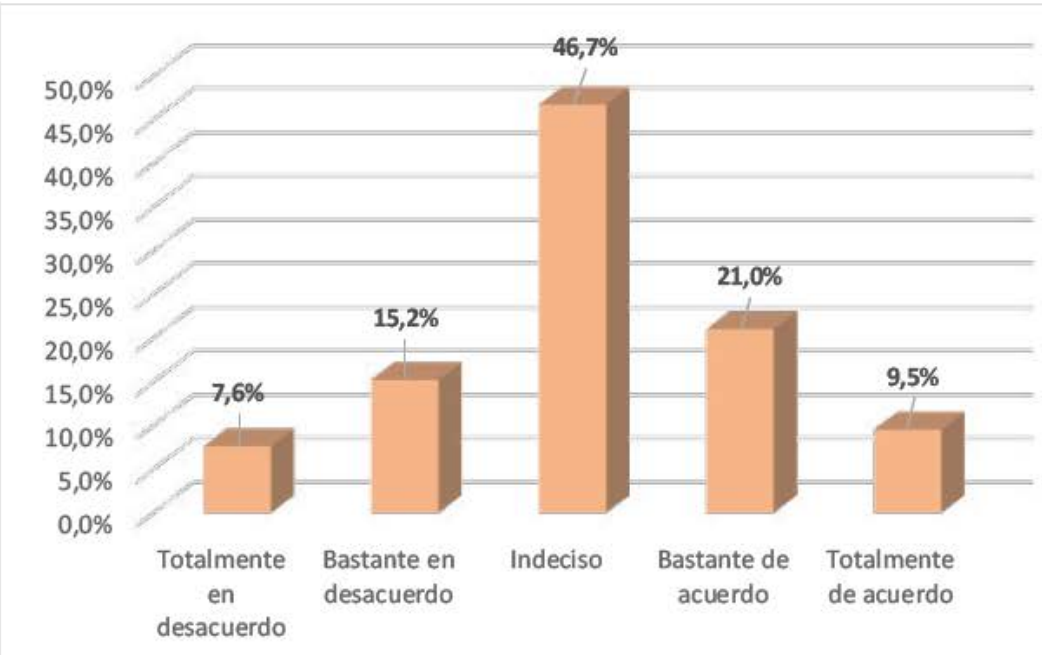

Figura 2. Grado de acuerdo sobre el buen rendimiento escolar del alumnado con altas capacidades. Fuente: Elaboración propia

En relación a los aspectos conductuales, el grado de acuerdo sobre si el alumnado con altas capacidades tiene problemas de conducta en el aula es compartido por la 
mayoría de los encuestados, un 61,9\%, estando en desacuerdo sólo el 12,4\%, tal y como queda reflejado en la Figura 3.

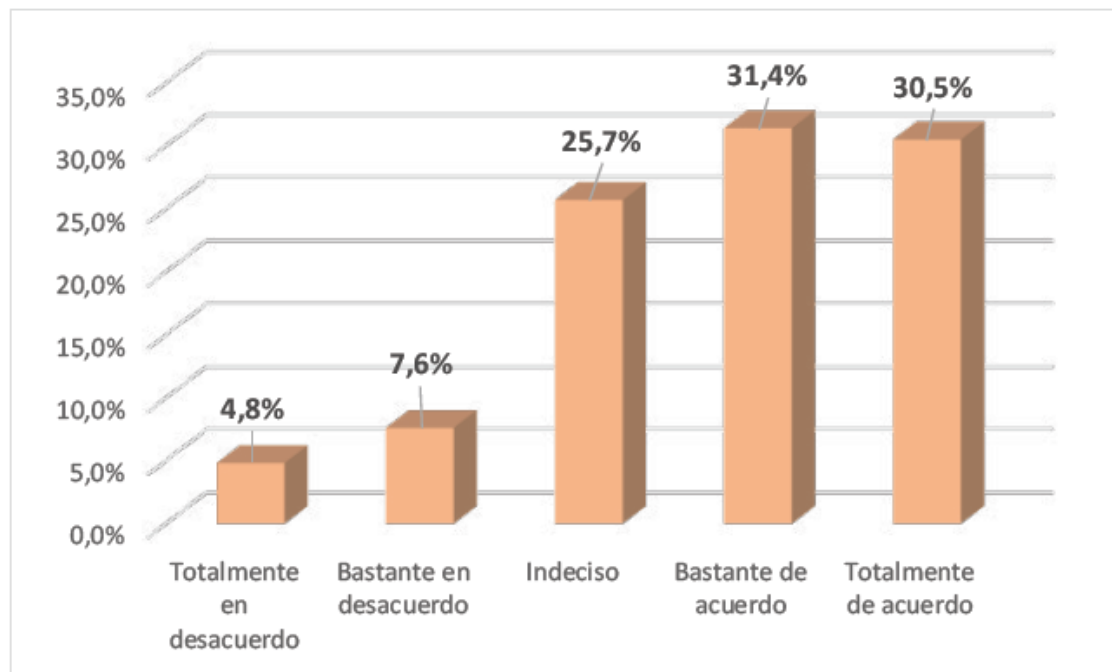

Figura 3. Grado de acuerdo sobre si los alumnos con altas capacidades pueden tener problemas de conducta en el aula. Fuente: Elaboración propia

Sobre cuál es la respuesta educativa más adecuada para este tipo de alumnado, se puede observar en la Figura 4, que el 80,9\% de los docentes considera que la educación del alumnado con altas capacidades intelectuales debe Ilevarse a cabo a través de programas de enriquecimiento siempre o casi siempre, siendo exigua la cantidad de docentes que consideran que no es una medida adecuada (3,9\%). Tanto la atención educativa en centros específicos como la realizada en aulas segregadas obtienen un amplio rechazo por los docentes encuestados.

Sobre los programas de aceleración como medida más adecuada para atender a este alumnado, el 52,3\% de los docentes encuestados la considera idónea, considerando que no se debe aplicar nunca o escasamente el 15,2\%. La inclusión con apoyos personalizados es considerada como una medida adecuada siempre o casi siempre por el $66,7 \%$ de los docentes encuestados, mientras que el $9,5 \%$ piensa que no debe llevarse a cabo nunca o escasamente.

Los resultados obtenidos a través del guion de entrevista formalizada en relación a la medida más adecuada para atender al alumnado con altas capacidades, cinco se muestran partidarios del enriquecimiento curricular sin ningún género de dudas, solo uno muestra como una opción a tener en cuenta la aceleración y únicamente uno de los docentes considera la aceleración como la medida más adecuada. 


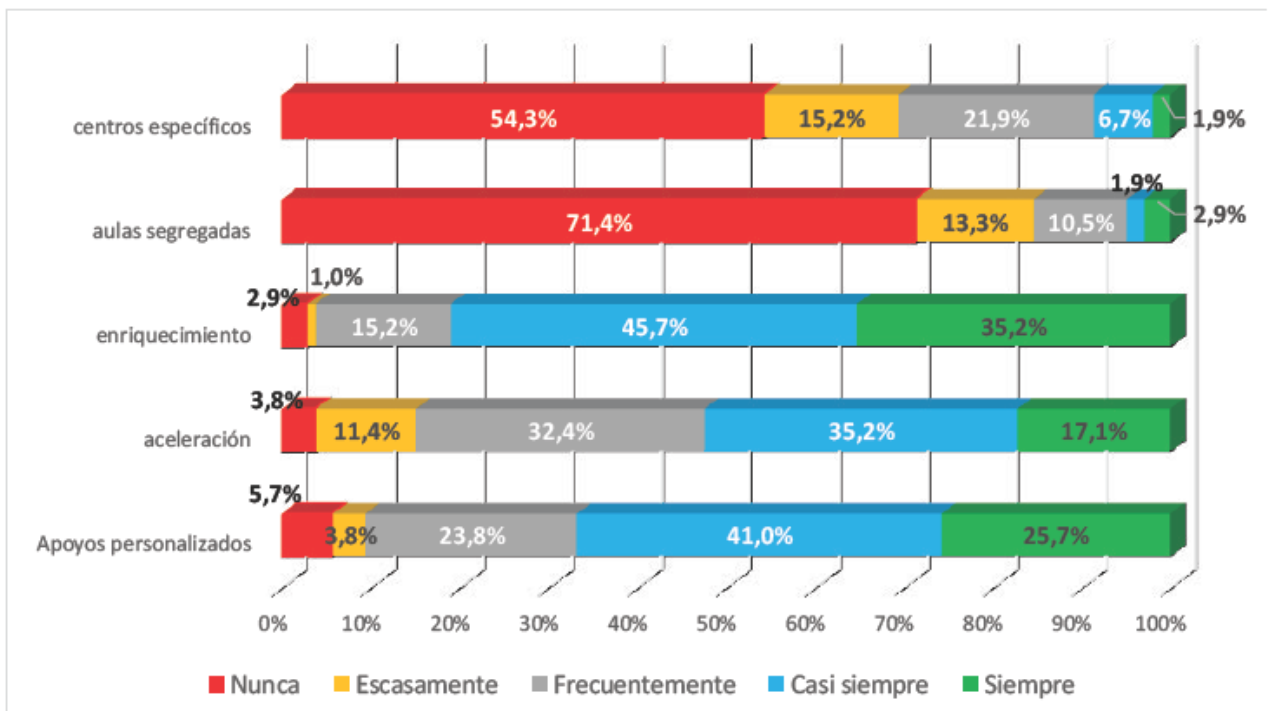

Figura 4. Preferencia de respuesta educativa al alumnado con altas capacidades. Fuente: Elaboración propia

Al ser demandados sobre cual es el motivo principal para declinarse por el enriquecimiento curricular frente a la aceleración, los docentes atribuían como el principal motivo, la evitación de la disincronía emocional, al desarrollarse el alumnado con altas capacidades acorde a su edad y con sus iguales. El docente que se declinó por la aceleración, consideró que la aceleración le proporcionaría a este alumnado la motivación que realmente necesita, sin que exista riesgo socioemocional, siempre que se lleve a cabo de forma adecuada.

\section{DisCUSIÓN Y CONCLUSIONES}

El hecho de que la gran mayoría de docentes consideren estar en desacuerdo con que los niños son más fáciles de diagnosticar que las niñas, es una percepción que es contraria a lo que establece Robison y Clikenbead (2008), Domínguez (2002) y Gómez (2018), ya que estos consideran que la presión social y el miedo a ser rechazadas por sus iguales hacen que las niñas camuflen sus altas capacidades para pasar inadvertidas (Domínguez, 2002; Peñas, 2006; Pérez, 2018),

Una de las percepciones de los docentes que suelen basarse en estereotipos y mitos, es la de relacionar a este tipo de alumnado con un buen rendimiento escolar. Los resultados obtenidos coinciden con esa visión estereotipada, ya que el 30,5\% de los docentes aún lo considera así, en contra de los que estableció Whitmore (1988), respecto a considerar como posible alumnado con altas capacidades también a los tenía bajo rendimiento escolar, rompiendo esa tradicional concepción de centrarse en los que tenían mejor rendimiento, cuestión que, a la vista de los resultados, todavía persiste. 
Esta creencia coincide con lo establecido por Pfeiffer (2015), Martínez y Guirado (2010) y Tourón Fernández y Reyero (2002), ya que algunos docentes, todavía se basan en sus procesos de detección de estos alumnos en concepciones tradicionales, principalmente en tener un alto rendimiento académico.

El hecho de que esta gran cantidad de docentes siga relacionando altas capacidades con alto rendimiento, explica otro factor causante de los bajos índices de diagnóstico, ya que choca frontalmente con lo que establece Cakir (2014), al afirmar que este tipo de alumnado puede presentar bajo rendimiento académico por la poca motivación en el entorno escolar, y con los que señala Wellisch y Brown (2012), cuando indica que la presencia del bajo rendimiento con características relacionadas con las altas capacidades es un síntoma de que el alumno tengas altas capacidades.

Otra de las percepciones basadas en estereotipos y mitos son las relacionadas con la conductas y comportamientos de este tipo de alumnado. Los resultados obtenidos, la gran mayoría, coinciden con lo establecido por Baudson y Preckel (2016), a percibir a este alumnado con menos habilidades sociales y menor capacidad de adaptación a su entorno que el resto del alumnado. Así mismo, también coinciden con las investigaciones de Baudson y Preckel (2013), en el que los docentes vinculan a este alumnado con la hipótesis de la falta de armonía, y por tanto, considerarles más inestables socioemocionalmente, incluso con los de Castro (2005) al considerarlos como elementos que influyen negativamente en el clima del aula, y los de Elices, Palazuelo y Caño, (2006) en donde los docentes creen que tienen problemas conductuales de forma habitual.

En relación sobre la medida más adecuada para la atención educativa de este alumnado, según los docentes, es la que se realiza a través de programas de enriquecimiento. Estos resultados coinciden con los de Sánchez Manzano (1997) y Reyero y Tourón (2003), pero no con los de González y Palomares (2016), en donde esta medida es la segunda más adecuada a juicio de los docentes, por detrás de los apoyos personalizados.

Según los docentes, y siempre en régimen de inclusión, la medida menos adecuada para atender a este alumnado es la aceleración o flexibilización que coincide con González y Palomares (2016), Reyero y Tourón (2003), Rodrigues y De Souza (2012), Carreras et al. (2015).

La razón aportada por los docentes para no considerar esta medida como la más adecuada es el riesgo socioemocional al que se somete el alumnado con altas capacidades. Esta percepción coincide plenamente con las investigaciones de Carreras et al. (2015), Jiménez et al. (2006).

En este sentido, la razón esgrimida por los docentes, de la supuesta disincronía emocional que padecería este alumnado al ser acelerado, no se corresponde con las consecuencias socioemocionales que la aceleración produce en este alumnado, según los estudios de Colangelo et al. (2004), Reyero y Tourón (2003), Sánchez Manzano (1997), Rodrigues y De Souza (2012), Wellisch y Brown (2012), Carreras et al. (2015), Jiménez et al. (2006) y Lee, Olszewski-Kubilius y Peternel (2010). 
En conclusión y relacionado con el objetivo general propuesto, se han conocido las percepciones de los maestros y maestras de Educación Primaria sobre aspectos fundamentales para el adecuado desarrollo del alumnado con altas capacidades. Se ha puesto de manifiesto a lo largo de este estudio, que los docentes son esenciales para que estos alumnos reciban la atención educativa que realmente necesitan, ya que de su actitud y buena práctica docente dependerá que estos alumnos sean identificados y logren desarrollar al máximo sus potencialidades.

Ha quedado evidenciado que la formación específica de los docentes en este ámbito es uno de los problemas que mayor repercusión tiene en el correcto desarroIlo de este alumnado. El problema no solo radica en la poca cantidad de docentes formados, sino en que la calidad de esa formación no es la óptima, lo que provoca que todavía una parte importante de los docentes de Educación Primaria conservan mitos y estereotipos que solo a través de una formación actualizada y de calidad se lograrían desterrar.

Por otro lado, esta deficiente formación de los docentes, implica también agudizar la problemática en cuanto a la detección e identificación de estos alumnos, lo que se traduce en que actualmente haya en las aulas de nuestra región una gran cantidad de niños y niñas con altas capacidades que no reciben la respuesta educativa que necesitan.

Por último, los docentes consideran como mejor medida para atender las necesidades de los alumnos con altas capacidades el enriquecimiento curricular, cuestión que es totalmente válida y en muchos casos acertada, pero el hecho de considerar que la aceleración es una medida poco recomendable, demuestra que existe una gran distancia entre la investigación y las aulas, ya que los supuestos riesgos socioemocionales que perciben los docentes no se encuentran completamente respaldados por la evidencia científica. Esta situación, hace que muchos alumnos con altas capacidades sí reciban la atención educativa que necesitan, siempre y cuando esta sea el enriquecimiento, pero otros muchos no la recibirán por esa creencia de proporcionar a todos los alumnos con altas capacidades la misma medida, sin considerar realmente si es la que más beneficio le reportará.

En definitiva, en el ámbito de las altas capacidades, los docentes pueden y deben realizar un proceso de transformación de la realidad, han de empoderarse para conseguir cambiar la tendencia de una práctica educativa que actualmente está lejos de satisfacer las necesidades educativas de este alumnado con altas capacidades intelectuales.

\section{REFERENCIAS BIBLIOGRÁFICAS}

Agudo, N. (2017). Un estudiante con altas capacidades en mi aula, ¿Ahora qué? Revista Nacional e Internacional de Educación Inclusiva, 10(1), 265-277. Recuperado de https://dialnet.unirioja.es/servlet/articulo?codigo $=6049220$.

Alonso, J. (2008). La educación de los alumnos con sobredotación intelectual. En J. Alonso (Ed.), Inteligencia, creatividad y talento: una inversión para la niñez en 
riesgo. VII Congreso Bienal de la Ficomundyt. Lima. pp. 37-64. Recuperado de http://www.templetonfellows.org/projects/docs/ficomundyt.pdf.

Baudson, T. y Preckel, F. (2013). Teachers' implicit personality theories about the gifted: An experiemtal approach. School Psychology Quarterly, 28(1), 37-46. https://doi.org/10.1037/spq0000011.

Baudson, T. y Preckel, F. (2016). Teachers' conceptions of gifted and average-Ability students on achievement-Relevant dimensions. Gifted Child Quarterly, 60(3), 212 225. https://doi.org/10.1177/0016986216647115.

Branda, S. y Porta, L. (2012). Maestros que marcan. Biografía personal e identidad profesional en docentes memorables. Revista de currículum y formación del profesorado, 16(3), 231-243. Recuperado de http://www.redalyc.org/ pdf/567/56725002012.pdf.

Cakir, L. (2014). The relationship between underachievement of gifted students and their attitudes toward school environment. Procedia - Social and Behavioral Sciences, 152, 1034-1038. https://doi.org/10.1016/j.sbspro.2014.09.269.

Calero, M. D. y García, M. B. (2011). La evaluación de los niños superdotados: ¿cuándo, por qué y cómo? Recuperado de http://www.infocop.es/view_article. asp?id=3304.

Carreras, L., García, C., Fernández, M., González, R., Sales, S. y Bernal, A. (2015). Estudio sobre la aceleración de curso como medida de intervención educativa para alumnos con altas capacidades (superdotados y talentos académicos). En M. Fernández y L. Carreras (Coords.), IV Jornadas Nacionales de Altas Capacidades. Educación y Creatividad. Barcelona. Recuperado de http://www.mentor.cat/ uploads/2/5/2/4/25246316/estudio_aceleraci\%C3\%B3n.pdf.

Carman, C. (2013). Comparación de manzanas y naranjas: quince años de definiciones de superdotación en la investigación. Revista de académicos avanzados, 24(1), 52-70. https://doi.org/10.1177/1932202X12472602.

Castro, M. (2005). Conocimiento y actitudes de maestros de Educación Infantil, Educación Primaria y estudiantes de Magisterio sobre los niños superdotados intelectualmente. (Tesis doctoral, Universidad Complutense de Madrid). Recuperado de http://biblioteca.ucm.es/tesis/psi/ucm-t28588.pdf.

Colangelo, N., Assouline, S. y Gross, M. (2004). Una nación engañada. lowa City: The Belin-Blank Center for Gifted Education. Recuperado de http://docs.wixstatic. com/ugd/b64a15_d062698e6fd34ddca94b36595314784d.pdf.

Domínguez, P. (2002). Sobredotación, mujer y sociedad. Faísca: revista de altas capacidades, 9, 3-34. Recuperado de https://dialnet.unirioja.es/servlet/ articulo?codigo=2476355.

Elices, J., Palazuelo, M. y Caño, M. (2006). El profesor, identificador de necesidades educativas asociadas a alta capacidad intelectual. Faisca. Revista de Altas Capacidades, 11(13), 23-47. https://doi.org/10.5209/rev_FAIS.2006.v11.n13.8624. 
Gómez, M. (2018, marzo 19). El silencio de las niñas y mujeres con Altas Capacidades [Entrada Blog]. Recuperado de http://www.altascapacidadesytalentos.com/ mujer-y-superdotacion/

González, R. y Palomares, A. (2016). Percepciones del profesorado sobre la inclusión educativa del alumnado con altas capacidades intelectuales. En A. Palomares (Coord.), Competencias y empoderamiento docente: Propuestas de investigación e innovación educativas en contextos inclusivos (pp. 117-122). Madrid: Síntesis.

Hernández, D. y Gutiérrez, M. (2014). El estudio de la alta capacidad intelectual en España: Análisis de la situación actual. Revista de Educación, 364, 251-272. https://doi.org/10.4438/1988-592X-RE-2014-364-261.

Hernández, R., Fernández, C. y Baptista, M. P. (2014). Metodología de la Investigación. ( $5^{\mathrm{a}}$ ed.). México D.F.: McGrawhill.

Jiménez, J., Artiles, C., Ramírez, G. y Álvarez, J. (2006). Evaluación de los efectos de la aceleración en alumnos con alta capacidad intelectual en la Comunidad Autónoma de Canarias. Infancia y Aprendizaje, 29(1), 51-64. Recuperado de http:// ejimenez.webs.ull.es/wp-content/uploads/2006-Evaluaci\%C3\%B3n-de-los-efectos-de-la-aceleraci\%C3\%B3n-en-alumnos-con-alta-capacidad-intelectual-en-laComunidad-Aut\%C3\%B3noma-de-Canarias.pdf.

Lee, S., Olszewski-Kubilius, P. y Peternel, G. (2010). The efficacy of academic acceleration for gifted minority students. Gifted Child Quarterly, 54(3), 189-208. http://doi. org/10.1177/0016986210369256.

Marland, S. P. (1972). Education of the gifted and talented: Report to the Congress of the United States by the U.S. Commisioner of Education. Washington: U.S. Government Printing Office. Recuperado de http://files.eric.ed.gov/fulltext/ ED056243.pdf.

Martínez, M. y Guirado, A. (2010). Alumnado con altas capacidades: Escuela Inclusiva: alumnos distintos, pero no diferentes. Barcelona: Graó.

Ministerio de Educación y Formación Profesional. Recuperado de http://www.mecd. gob.es/servicios-al-ciudadano-mecd/estadisticas/educacion/no-universitaria/profesorado/estadistica.html.

Muglia, S. y Tonete, J. (2016). Percepção de professores em cursos de formação sobre talentos/superdotação. Revista de Psicología (PUCP), 34(1), 29-60. Recuperado de http://www.scielo.org.pe/scielo.php?script=sci_isoref\&pid=S025492472016000100003\&lng=es\&tlng=pt

Nolla, C. (2012). Análisis y valoración de la situación actual del alumnado con altas capacidades en España. Revista Educación Inclusiva, 5(2), 129-139. Recuperado de http://revistaeducacioninclusiva.es/index.php/REl/article/view/238/232.

Palella, S. y Martins, F. (2012). Metodología de la investigación cuantitativa. Caracas. Fedupel. Recuperado de http://es.calameo.com/read/000628576f51732890350. 
Peña del Agua, A., Martínez, R., Velázquez, A., Barriales, M. y López, L. (2003). Estudio de las características que percibe el profesorado en alumnos con alta capacidad intelectual. Revista de Investigación Educativa, 21(1), pp. 271-289. Recuperado de http://revistas.um.es/rie/article/view/99211/94811.

Peñas, M. (2006). Características socioemocionales de las personas adolescentes superdotadas. Ajuste psicológico y negación de la superdotación en el concepto de sí mismas (Tesis doctoral. Universidad Pontificia Comillas, Madrid). Recuperado de https://dialnet.unirioja.es/servlet/tesis?codigo=105906.

Pérez, L. (2018, marzo 19). El silencio de las niñas y mujeres con Alta Capacidades [Entrada Blog]. Recuperado de http://www.altascapacidadesytalentos.com/mujery-superdotacion/.

Pfeiffer, S. I. (2015). The tripartite model on high capacity and best practices in the evaluation of the ablest. Revista Educación, 368, 66-95. https://doi.org/10.4438/1988592X-RE-2015-368-293.

Reis, S. y Renzulli, J. (2010). Is there still a need for gifted education? An examination of current research. Learning and Individual Differences, 20(4), 308-317. https:// doi.org/10.1016/j.lindif.2009.10.012.

Reyero, M. y Tourón, J. (2003). El desarrollo del Talento. La Aceleración como Estrategia Educativa. A Coruña: Netbiblo. Recuperado de https://es.scribd.com/document/256133667/El-Desarrollo-Del-Talento-Libro\#download\&from_embed

Robinson, A. y Clinkeanbeard, P. R. (2008). History of giftedness: Perspectives from the past presage modern scholarship. En S. I. Pfeiffer (Ed.), Handbook of giftedness in children (pp. 13-31). New York: Springer. Recuperado de http://www.umpalangkaraya.ac.id/dosen/dwisariusop/wp-content/uploads/2016/11/Handbook-ofgiftedness-in-children.pdf \#page $=273$

Rodrigues, R. y De Souza, D. (2012). Aceleración de la enseñanza para alumnos superdotados: argumentos favorables y contrarios. Revista de Psicología, 30(1), 190-214. Recuperado de http://revistas.pucp.edu.pe/index.php/psicologia/article/ view/2634/2581.

Sánchez Manzano, E. (1997). Hacia una didáctica para la educación de los niños superdotados. Revista Complutense de Educación, 8(2), 57-70. https://doi. org/10.5209/RCED.18287.

Sánchez Manzano, E. (2009). La superdotación intelectual. Málaga: Aljibe. Recuperado de http://site.ebrary.com/lib/bibliotecauclm/detail.action?docID=11217180.

Sánchez Manzano, E. (2010). El modelo tetrártico de superdotación emergente. Revista de Psicología y Educación, 1(5), 51-68. Recuperado de http://www.revistadepsicologiayeducacion.es/pdf/46.pdf.

Tourón, J. (2015, mayo 15). ¿Conoces el modelo tetrártico sobre la alta capacidad? [Entrada blog]. Recuperado de https://www.javiertouron.es/conoces-el-modelotripartito-sobre-la/. 
Tourón, J., Fernández, R. y Reyero, M. (2002). Actitudes del profesorado hacia la superdotación. Implicaciones para el desarrollo de programas de formación. Faisca. Revista de Altas Capacidades, 9, 95-110. https://doi.org/10.5209/rev_FAIS.2002. v9.8674.

Wellisch M. y Brown J. (2012). An integrated identification and intervention model for intellectually gifted children. Journal of Advanced Academics, 23(2), 145-167. https://doi.org/10.1177/1932202X12438877.

Whitmore, J. (1988). Nuevos retos a los métodos de identificación habituales. En Freeman, J., Los niños superdotados: aspectos pedagógicos y psicológicos. Madrid: Santillana. 